\title{
Excitation of $\mathrm{O}_{2}{ }^{1} \Delta_{\mathrm{g}}$ by Electron Impact*
}

\author{
P. S. Julienne and M. Krauss \\ Institute for Materials Research, National Bureau of Standards, Washington, D.C. 20234
}

(June 21, 1972)

\begin{abstract}
The total and differential cross sections as a function of energy were calculated for electron impact excitation of the $\mathrm{O}_{2}{ }^{1} \Delta_{g}$ state from the ground ${ }^{3} \Sigma_{\bar{g}}$ state. The Ochkur-Rudge approximation was used for the exchange amplitude, which was calculated using Hartree-Fock. wave functions with a Gaussian basis. There is good agreement with the experimental total cross section except near threshold.
\end{abstract}

Key words: Ab initio Gaussian wave functions; electron impact excitation; exchange amplitude; $\mathrm{O}_{2}{ }^{1} \Delta_{g}$ state; Ochkur-Rudge approximation.

Optically forbidden molecular transitions involving a change in electron spin may be excited by electron impact through electron exchange. Such processes are particularly of interest for common atmospheric molecules. We have calculated total and differential cross sections for the excitation of the ${ }^{1} \Delta_{g}$ state of $\mathrm{O}_{2}$ from the ground ${ }^{3} \Sigma_{g}$ state using the OchkurRudge approximation $[1,2,3]$. This is essentially a first order Born approximation for the exchange amplitude in which distortion of the incident electron wave function and polarization of the molecule are neglected. However, in calculations of excitation cross sections for triplet states in $\mathrm{H}_{2}$ [4] and for the $\mathrm{B}^{3} \Pi_{g}$ state of $\mathrm{N}_{2}[5,6]$ this approximation gives reasonable agreement with experimental total cross sections. This report summarizes the results of our calculation of the $\mathrm{O}_{2}{ }^{1} \Delta_{g}$ cross section and makes a comparison with experimental data $[7,8,9]$.

In the Ochkur-Rudge approximation the differential cross section for excitation from a state $i$ to a state $f$ is given in atomic units $\left(a_{0}^{2} / s r\right)$ by the following expression

$$
I(\theta)=4 \frac{g_{f}}{g_{i}} \frac{K_{f}}{K_{i}} \frac{1}{T^{4}}|A|^{2}
$$

where $g_{i}, g_{f}$ are degeneracy factors, $K_{i}$ and $K_{f}$ the magnitudes in atomic units $\left(a_{0}^{-1}\right)$ of the initial and final wave vectors, and $T$ and $A$ are defined by

$$
\begin{gathered}
T=\sqrt{K_{i}^{2}+\epsilon} \\
A(K)=\int \psi_{f}^{*} \psi_{i} e^{i K \cdot r} d \mathbf{r} .
\end{gathered}
$$

Here $\epsilon$ is the ionization potential of the initial state in Rydbergs and

\footnotetext{
* This work was supported by the Naval Research Laboratory, Washington, D.C.
}

$$
\mathbf{K}=\mathbf{K}_{f}-\mathbf{K}_{i}
$$

is the momentum transfer vector having magnitude

$$
K=\sqrt{K_{i}^{2}+K_{f}^{2}-2 K_{i} K_{f} \cos \theta^{\prime}}
$$

where $\theta^{\prime}=\pi-\theta$ is the angle between $K_{i}$ and $K_{f}$. The total cross section

$$
\sigma=2 \pi \int_{0}^{\pi} I(\theta) \sin \theta d \theta
$$

is expressed in terms of an integration over $K$ as

$$
\sigma=\frac{4}{K_{i}^{2} T^{4}} \frac{g_{f}}{g_{i}} \int_{K_{\min }}^{K_{\max }}|A(K)|^{2} K d K
$$

where $K_{\min }=K_{f}-K_{i}$ and $K_{\max }=K_{f}+K_{i}$.

For molecular transitions the wave functions $\psi_{i}$ and $\psi_{f}$ are total wave functions including the vibrational and rotational motion. However, we wish to consider here only the cross section averaged over initial and final rotational and vibrational states, and therefore calculate $A(K)$ at the equilibrium position of the nuclei. This is reasonable for the $\mathrm{O}_{2} \mathrm{X}^{3} \Sigma_{g}^{-}-a^{1} \Delta_{g}$ transition since the $0-0$ Franck-Condon factor is 0.986 [7].

The dominant single configuration representing the molecular oxygen $\mathrm{X}^{3} \Sigma_{\bar{g}}$ and $\mathrm{A}^{1} \Delta_{g}$ states is

$$
1 \sigma_{g}^{2} 1 \sigma_{u}^{2} 2 \sigma_{g}^{2} 2 \sigma_{u}^{2} 3 \sigma_{a}^{2} 1 \pi_{u}^{4} 1 \pi_{g}^{2}
$$

with $1 \pi_{g}^{2}$ coupled ${ }^{3} \Sigma$ - in the former and ${ }^{1} \Delta$ in the latter. The transition is possible since the incident electron can couple with each of these states to produce a 
doublet. A detailed consideration of these antisymmetrized doublet wave functions show that the matrix element A may be written as

$$
|A|=\frac{3}{\sqrt{6}}<1 \pi_{g}^{y}(\Sigma)\left|e^{i \mathbf{K} \cdot \mathbf{r}}\right| 1 \pi_{g}^{x}(\Delta)>
$$

where $\pi^{ \pm 1}=\left(\pi^{x} \pm i \pi^{y}\right) / \sqrt{2}$. This matrix element was evaluated analytically using a Gaussian expansion basis for the $1 \pi_{g}$ orbitals, which were determined by a Hartree-Fock self-consistent field procedure [10]. The Gaussian basis, which is derived from the atom results of Huzinaga [11], contained a total of 48 functions and should represent the orbitals well. A subsequent multi-configuration self-consistent field calculation using a Slater trial orbital expansion basis [12] indicates that neglecting electron correlation near $R_{e}$ probably does not introduce an error in $A$ greater than 20 percent or so.

$A(K)$ was evaluated between $K=0$ and $5 a_{0}^{-1}$ at equal intervals of $0.25 a_{0}^{-1}$. This, plus an accurate numerical interpolation formula, permitted the evaluation of $I(\theta)$ and $\sigma$ for a number of incident electron energies. The results for the total cross section are shown in figure 1 , which also includes the experimental points of Trajmar et al. [7]. As may be expected for a Born-type approximation, the high energy tail is calculated fairly well, whereas the low energy calculated cross sections differ appreciably from the experimental values. The source of this difference is clear when one examines the differential cross section. The present approximation necessarily predicts no forward peaking in $I(\theta)$, whereas Trajmar et al., observe strong forward peaking, as shown in figure 2. The major difference between the calculated and experimental total cross sections below $10 \mathrm{eV}$ results from the difference in the small angle differential cross section. (However, the contribution to

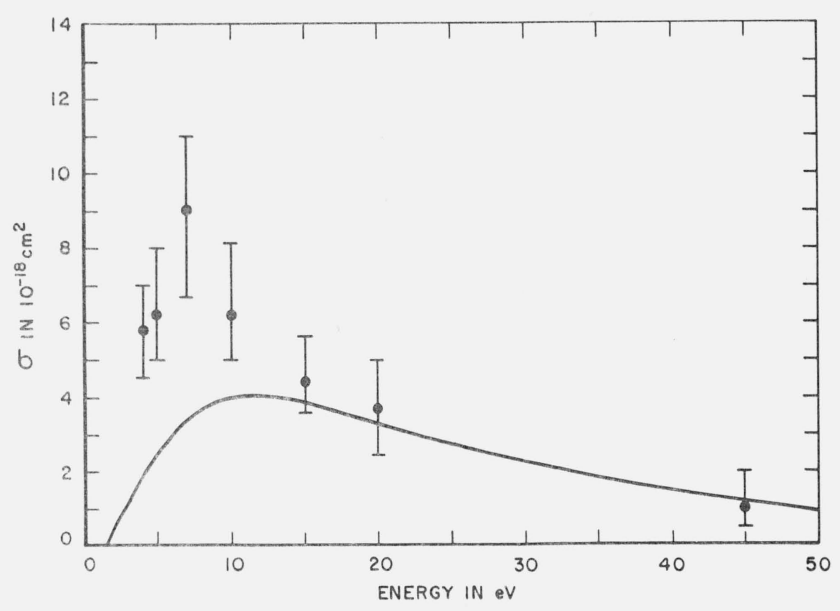

FIGURE 1. Calculated total cross section for excitation of ${ }^{1} \Delta_{\mathrm{g}}$ as a function of incident electron energy.

The experimental points of Trajmar et al., are also shown.

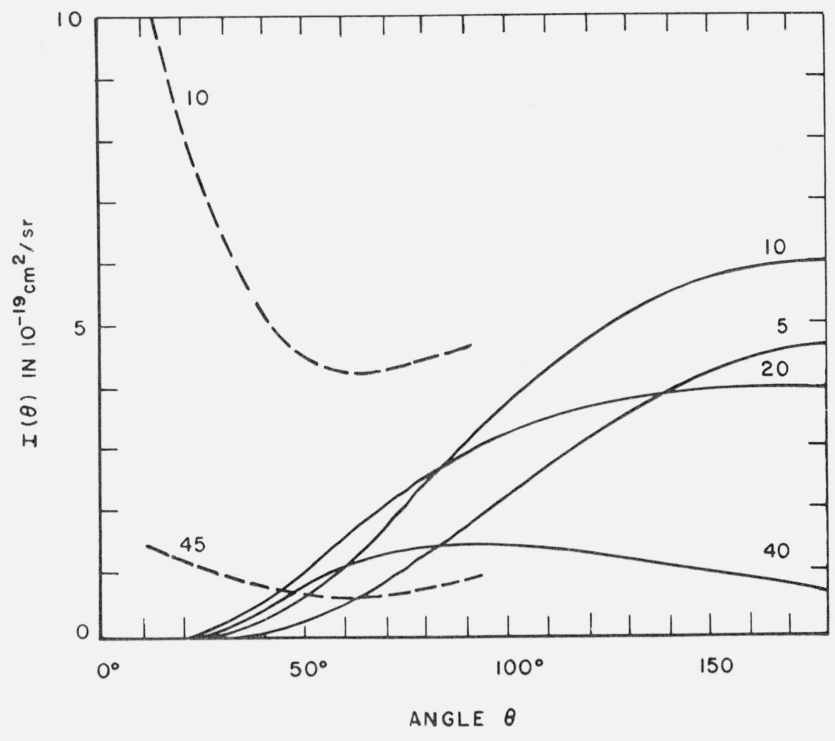

FIGURE 2. Calculated differential cross section for excitation of ${ }^{1} \Delta_{g}$ for incident electron energies of $5,10,20$, and $40 \mathrm{eV}$.

Experimental values of Trajmar et al., at 10 and $45 \mathrm{eV}$ are also shown.

the total cross section from forward peaking is somewhat reduced by the volume element $\sin \theta d \theta$ ).

The total cross section has also been measured above $20 \mathrm{~V}$ by Konishi et al. [9]. Although their values differ appreciably from those of Trajmar et al., the latter authors point out that there is not necessarily any inconsistency between the two results if the error limits on both measurements are taken into consideration. The differential cross section has been measured at an incident energy of $30 \mathrm{eV}$ by Suzuki et al. [8]. Their large angle cross section does not differ from the present result or that of Trajmar et al. (estimated by interpolation) by more than a factor of 2 or so. However, they do not observe the forward peaking of the latter authors. Their cross section for angles less than about $30^{\circ}$ is decreasing and appears to be tending towards zero.

The large peak in the total cross section around $7 \mathrm{eV}$ has been ascribed [13] to scattering through excited states of $\mathrm{O}_{2}$. Only the ${ }^{2} \Pi_{u}$ state can contribute by a first-order exchange mechanism. This state can also decay by dissociation to $\mathrm{O}^{(3 \mathrm{P})}+\mathrm{O}^{-}\left({ }^{2} \mathrm{P}\right)$ and back to the ground state of $\mathrm{O}_{2}$. Since the ${ }^{2} \Pi_{u}$ state is expected to have a small electronic width, it is not certain that this resonance state contributes substantially to the forward peaking and total cross section at low energies. But this mechanism shows how inaccurate the OchkurRudge approximation can be at low energies.

This simple approximation appears to provide a useful estimate of the total cross section for the exchange scattering as observed by all who have applied it. At the equilibrium separation the Gaussian basis SCF wave functions also appear to be sufficiently accurate as long as the inherent approximate nature of the method is realized. 


\section{References}

[1] Ochkur, V. I., Zh. Eksp. Teor. Fiz. 45, 734 (1963) [Sov. Phys. JETP 18, 503 (1964)].

[2] Rudge, M. R. H., Proc. Phys. Soc. (London) 85, 607 (1965); 86, 763 (1965).

[3] Truhlar, D. G., Cartwright, D. C., Kuppermann, A., Phys. Rev. 175, 113 (1968)

[4] Cartwright, D. C., Kuppermann, A., Phys. Rev. 163, 86 (1967).

[5] Chung, S., Lin, C. C., Ap. Optics 10, 1790 (1971).

[6] Cartwright, D. C., Phys. Rev. A2, 1331 (1970).

[7] Trajmar, S., Cartwright, D. C., Williams, W., Phys. Rev. A4, 1482 (1971).

[8] Suzuki, H., Wakiya, K., Takayanagi, T., Okino, T., Studies of
Atomic Collisions and Related Problems in Japan. Progress Report, Institute of Space and Aeronautical Science, University of Tokyo, March 1971, p. 8.

[9] Konishi, A., Yamamoto, K. W., Suzuki, H. J. Phys. Soc. Japan 29, 526 (1970).

[10] The present calculations were made with a computer program IBMOL2, that was written by E. Clementi and A. Veillard, and modified to suit the open-shell problem. See E. Clementi and D. R. Davis. I. Comput. Phys. 1, 223 (1966).

[11] Huzinaga, S., J. Chem. Phys. 42, 1293 (1965).

[12] Julienne P. S., and Krauss, M., unpublished.

[13] Larkin, I. W., and Hasted, J. B., J. Phys. B5, 95 (1972).

(Paper 76A6-750) 